\title{
EMPIRICAL LAPLACE TRANSFORM AND APPROXIMATION OF COMPOUND DISTRIBUTIONS
}

\author{
by \\ Sándor Csörgö ${ }^{1}$, Szeged University, Hungary \\ Jef L. Teugels, Katholieke Universiteit Leuven, Belgium
}

\section{SUMMARY}

Let $\left\{\mathrm{X}_{\mathrm{i}}\right\}_{1}^{\infty}$ be i.i.d. non-negative r.v.'s with d.f. $\mathrm{F}$ and Laplace transform L. Let $\mathrm{N}$ be integer valued and independent of $\left\{\mathrm{X}_{\mathrm{i}}\right\}_{1}^{\infty}$. In many applications one knows that for $\mathrm{y} \rightarrow \infty$ and a function $\varphi$

$$
\mathrm{P}\left\{\sum_{\mathrm{i}=1}^{N} \mathrm{X}_{\mathrm{i}}>\mathrm{y}\right\} \sim \varphi\left(\mathrm{y}, \tau, \mathrm{L}(\tau), \mathrm{L}^{\prime}(\tau), \ldots\right)
$$

where in turn $\tau$ is the solution of an equation

$$
\psi(\tau, \mathrm{L}(\tau), \ldots)=0
$$

On the basis of a sample of size $n$ we derive an estimator $\tau_{n}$ for $\tau$ by solving $\psi\left(\tau_{n}, L_{n}\left(\tau_{n}\right), L_{n}^{\prime}\left(\tau_{n}\right), \ldots\right)=0$ where $L_{n}$ is the empirical version of $L$. This estimator is then used to derive the asymptotic behaviour of $\varphi\left(y, \tau_{n}, L_{n}\left(\tau_{n}\right), L_{n}^{\prime}\left(\tau_{n}\right), \ldots\right)$. We include five examples, some of which are taken from insurance mathematics.

Keywords : Empirical Laplace transform, compound distribution, probability of ruin. MOS-classification : 60E10, 62F10, 62P05

1Work partially supported by the Hungarian National Foundation for Scientific Research, Grants $1808 / 86$ and $457 / 88$. 


\section{GENERAL PROCEDURE}

Let $X$ be a non-degenerate random variable with d.f. $F$ on $[0, \infty)$ and with Laplace transform

$$
L(s):=E\left(e^{-s X}\right)=\int_{0}^{\infty} e^{-s x} d F(x)
$$

We always assume that $L(s)$ exists in an open neighbourhood of the origin; let $-\sigma$ be the abscissa of convergence of $L(s)$ and put $I=(-\sigma, \infty)$ or $I=[-\sigma, \infty)$ according to the case where $\mathrm{L}(\mathrm{s})$ converges at $-\sigma$ or not respectively. The function $\mathrm{L}($.$) is arbitrarily many$ times differentiable in the interior of $I$ and since $X \geq 0$ it is a decreasing convex function on $I$. Let int $I$ be the interior of $I$.

Many applications are concerned with compound distributions for which an approximation is needed. This approximation contains ingredients such as $\mathrm{L}(\mathrm{s})$ and derivatives at a point $\mathrm{s}=\tau$ where $\tau$ is a proper solution of an equation involving in turn $\mathrm{L}(\mathrm{s})$ and/or some of its derivatives. Saddle-point type approximations for nonnegative r.v.-s provide a good general example.

In practical situations one often doesn't know the precise form of $\mathrm{L}(\cdot)$; one only has a sample version $L_{n}(\cdot)$ based on a sample of independent observations $\mathrm{X}_{1}, \mathrm{X}_{2}, \ldots, \mathrm{X}_{\mathrm{n}}$ of $\mathrm{X}$. Hence

$$
L_{n}(s)=\frac{1}{n} \sum_{j=1}^{n} e^{-s X} j=\int_{0}^{\infty} e^{-s x} d F_{n}(x)
$$

where $F_{n}(x)=\frac{1}{n} \#\left\{1 \leq j \leq n: X_{j} \leq x\right\}$ is the empirical distribution function of the sample. This empirical Laplace transform $\mathrm{L}_{\mathbf{n}}(\mathrm{s})$ is a random analytic function for all (complex) values of 8 . A natural sample-based estimator of the approximation will be obtained by replacing the derivatives of $L$ by those of $L_{n}$ both in the approximation and in the equation defining $\tau$, the latter will then automatically define an estimator $\tau_{n}$ for $\tau$.

An adaption of the proof of the Proposition in Csörgö (1982) easily yields the following auxiliary result, where $L^{(k)}$ is the $k$-th derivative of $L, k=0,1,2, \ldots$. 
Proposition 1. If $\mathrm{J}$ is any closed interval contained in $\mathrm{I}$, then almost surely as $\mathrm{n} \rightarrow \infty$ for any bounded $k \in \mathbb{N}$

$$
\sup _{s \in J}\left|L_{n}^{(k)}(s)-L^{(k)}(s)\right| \rightarrow 0
$$

This proposition and some elementary analysis imply a crucial consistency result in that $\tau_{\mathbf{n}} \rightarrow \tau$ almost surely whenever $\tau \in$ int $\mathbf{I}$.

A more elaborate analysis, based on the defining relation for $\tau$ and $\tau_{n}$, will provide us with a limit in distribution for $\sqrt{n}\left(\tau_{n}-\tau\right)$. The limit in distribution whenever it exists - will depend on the limits in distribution of some auxiliary r.v.'s that we introduce here for later reference.

For any $s \in$ int $I$ we define

$$
W_{k, n}(s):=\frac{1}{\sqrt{n}} \sum_{j=1}^{n}\left\{X_{j}^{k} e^{-s X_{j}}-E\left(X^{k} e^{-s X}\right)\right\}
$$

Then it follows from the central limit theorem that

$$
\mathrm{W}_{\mathrm{k}, \mathrm{n}}(\mathrm{s}) \underset{\mathrm{n} \rightarrow \infty}{\mathfrak{D}} \mathfrak{N}\left(0, \sigma_{\mathbf{k}}^{2}(\mathrm{~s})\right)
$$

where $\stackrel{\mathfrak{D}}{\longrightarrow}$ denotes convergence in distribution and

$$
\begin{aligned}
\sigma_{k}^{2}(s) & =\operatorname{Var}\left(X^{k} e^{-s X}\right) \\
& =E\left[X^{2 k} e^{-2 s X}\right]-E^{2}\left(X^{k} e^{-s X}\right) \\
& =(-2)^{-2 k}{ }_{L}(2 k)(2 s)-\left(L^{(k)}(s)\right)^{2}
\end{aligned}
$$

The latter result only holds if $2 s \in$ int $I$. For later use we quote the next result.

Proposition 2. If $2 \mathrm{~s} \in$ int $I$ then the r.v.'s defined by (3) satisfy

$$
W_{k, n}(s) \underset{n \rightarrow \infty}{\mathcal{D}} \mathfrak{N}\left(0, \sigma_{k}^{2}(s)\right)
$$


where $\sigma_{k}^{2}(s)$ is given by (4).

In many applications we need variables of the type (3) but with $s$ replaced by a random sequence $s_{n}$ converging almost surely to $s$. A one term Taylor expansion of the type

$$
e^{-s_{n} X_{j}}=e^{-s X_{j}}-\left(s_{n}-s\right) X_{j} e^{-s}(j) X_{j}
$$

introduces random quantities $s_{n}(j)$ satisfying the inequalities

$$
\min \left(s_{n}, s\right) \leq s_{n}(j) \leq \max \left(s_{n}, s\right)
$$

We will use the following abbreviations

$$
\left\{\begin{array}{l}
S_{k, n}(s)=\frac{1}{n} \sum_{j=1}^{n} X_{j}^{k} e^{-s X_{j}}=(-1)^{k_{L}(k)}(s) \\
S_{k, n}\left(s_{n}, s\right)=\frac{1}{n} \sum_{j=1}^{n} X_{j}^{k} e^{-s}(j) X_{j}
\end{array}\right.
$$

where $s_{n}(j)$ is determined by $(6)$ and $(7)$.

Combining a strong law of large numbers with proposition 1 we obtain a useful result.

Proposition 3. Let $s \in$ int $I$; let $s_{n}+s$ almost surely. Then for any bounded $k \in \mathbb{M}$ almost surely as $n \rightarrow \infty$

(i) $s_{k, n}(s) \rightarrow E\left[X^{k} e^{-s X}\right]$

(ii) $S_{k, n}\left(s_{n}\right) \rightarrow E\left[X^{k} e^{-s X_{1}}\right]$

(iii) $S_{k, n}\left(s_{n}, s\right) \rightarrow E\left[X^{k} e^{-s X}\right]$

For easy reference we quote some Slutsky-type results (Serfling (1980, p. 19)), where c is a constant. 
Proposition 4. (i) $\mathrm{X}_{\mathrm{n}} \stackrel{\mathfrak{D}}{\rightarrow} \mathrm{X}, \mathrm{Y}_{\mathrm{n}} \stackrel{\mathfrak{D}}{\rightarrow} \mathrm{c} \Rightarrow \mathrm{X}_{\mathrm{n}}+\mathrm{Y}_{\mathrm{n}} \stackrel{\mathfrak{D}}{\rightarrow} \mathrm{X}+\mathrm{c}$

(ii) $X_{n} \stackrel{\mathfrak{D}}{\rightarrow} X, Y_{n} \stackrel{\mathfrak{D}}{\rightarrow} c \Rightarrow X_{n} Y_{n} \stackrel{\mathfrak{D}}{\rightarrow} X c$.

We now apply the above procedure to a number of specific examples. After shortly introducing the example we then give subsequently

(i) general observations concerning the solution $\tau$

(ii) the asymptotic behaviour of $\sqrt{n}\left(\tau_{n}-\tau\right)$

(iii) the asymptotic behaviour of the estimator for the required approximation.

We always assume $\sigma>0$, and $2 \tau \in$ int I whenever necessary.

\section{COMPOUND PASCAL DISTRIBUTION}

Assume $Y=\sum_{i=1}^{N} X_{i}$ where $P[N=n]=\left(\begin{array}{c}r+n-1 \\ n\end{array}\right) p^{r} q^{n}$ and where $N$ and $\left\{X_{j}\right\}_{1}^{\infty}$ are independent; further, $\mathrm{r} \in \mathbb{N}, 0<\mathrm{p} .<1, \mathrm{q}=1-\mathrm{p}$. Then $\mathrm{Y}$ has a compound Pascal distribution

$$
P\{Y \leq y\}=\sum_{n=0}^{\infty}\left(\begin{array}{c}
r+n-1 \\
n
\end{array}\right) p^{r} q^{n} F^{*} n(y)
$$

where $F^{*} n$ is the $n$-fold convolution of $F$.

It has been known for a long time that under our assumptions on $\mathrm{L}(\mathrm{s})$; i.e. $\sigma>0$, the tail $\mathrm{P}[\mathrm{Y}>\mathrm{y}]$ has a gamma type approximation. See Beekman (1974, p. 66). In Teugels (1985) and Embrechts e.a. (1985) it is shown that more precisely

$$
\mathrm{P}[\mathrm{Y}>\mathrm{y}] \sim \frac{\mathrm{e}^{\tau \mathrm{y}_{\mathrm{p}} \mathrm{y}^{\mathrm{r}-1}}}{\Gamma(\mathrm{r})|\tau|\left(\mathrm{L}^{\prime}(\tau)\right)^{\mathrm{r}}}, \mathrm{y} \rightarrow \infty
$$

where $L(\tau)=\frac{1}{q}$. See also Sundt (1982).

(i) First note that as $q<1, \tau<0$. To have a proper solution within int I we need $L(-\sigma)>\frac{1}{q}$. 
(ii) We will estimate $\tau$ by solving $L_{n}\left(\tau_{n}\right)=\frac{1}{q}$. Now $\tau_{n} \rightarrow \tau$ a.s. Furthermore

$$
\begin{aligned}
0 & =n\left(L_{n}\left(\tau_{n}\right)-L(\tau)\right) \\
& =\sum_{j=1}^{n}\left(e^{-\tau} X_{j}-E\left(e^{-\tau X}\right)\right)
\end{aligned}
$$

and by (6) and (7)

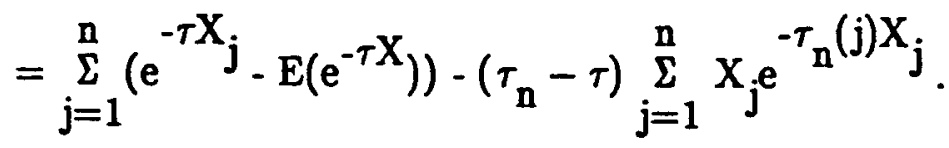

Hence

$$
\sqrt{n}\left(\tau_{\mathrm{n}}-\tau\right)=\frac{\mathrm{V}_{0, \mathrm{n}}(\tau)}{\mathrm{S}_{1, \mathrm{n}}\left(\tau_{\mathrm{n}}, \tau\right)}
$$

By prop. 2 and $3\left(\right.$ iii) $\sqrt{n}\left(\tau_{n}-\tau\right) \stackrel{\mathcal{D}}{\longrightarrow} \mathfrak{N}\left(0, \gamma^{2}\right)$ where $\gamma^{2}=\sigma_{0}^{2}(\tau) / \mathrm{E}^{2}\left[\mathrm{Xe}^{-\tau \mathrm{X}}\right]$ if $2 \tau \in$ int I.

The quantity $\gamma$ can be consistently estimated by

$$
\begin{aligned}
\gamma_{n}:= & \frac{1}{-L_{n}^{\prime}\left(\tau_{n}\right)}\left\{L_{n}\left(2 \tau_{n}\right)-L_{n}^{2}\left(\tau_{n}\right)\right\}^{1 / 2} \\
& =\frac{\left[S_{0, n}\left(2 \tau_{n}\right)-q^{-2}\right]^{1 / 2}}{S_{1, n}\left(\tau_{n}\right)}
\end{aligned}
$$

in view of proposition 3 ; hence almost surely $\gamma_{\mathbf{n}} \rightarrow \boldsymbol{\gamma}$.

With denoting the standard normal distribution function, let $z_{\alpha / 2}$ be the percentile point for which $\\left(z_{\alpha / 2}\right)=1-\frac{\alpha}{2}, 0<\alpha<1$. Then by the above construction we get an asymptotic confidence interval for $\tau$ if $2 \tau \in$ int $I$ :

$$
\lim _{n \rightarrow \infty} P\left\{\tau_{n}-z_{\alpha / 2} \frac{\gamma_{n}}{\sqrt{n}} \leq \tau \leq \tau_{n}+z_{\alpha / 2} \frac{\gamma_{n}}{\sqrt{n}}\right\}=1-\alpha .
$$


(iii) The approximation (9) of $\mathrm{P}[\mathrm{Y}>\mathrm{y}]$ depends upon $\mathrm{C}:=\frac{\mathrm{e}^{\tau \mathrm{y}}}{|\tau|\left|L^{\prime}(\tau)\right|^{\mathrm{r}}}$ which is itself estimated by

$$
C_{n}:=\frac{e^{\tau_{n} y}}{\left|\tau_{n}\right|\left|L_{n}^{\prime}\left(\tau_{n}\right)\right|^{r}}
$$

From proposition 1 and standard arguments $C_{n}+C$ almost surely. We should like to find the limiting distribution of $\sqrt{n}\left(C_{n}-C\right)$. Now

$$
C_{n}-C=\frac{e^{\tau y}}{\tau\left(-L^{\prime}(\tau)\right)^{\Gamma}}-\frac{e^{\tau_{n} y}}{\tau_{n}\left(-L_{n}^{\prime}\left(\tau_{n}\right)\right)^{I}}
$$

or

$$
e^{-\tau y} \tau \cdot \tau_{n}\left(L^{\prime}(\tau) L_{n}^{\prime}\left(\tau_{n}\right)\right)^{r}\left(C_{n}-C\right)=\tau_{n}\left(-L_{n}^{\prime}\left(\tau_{n}\right)\right)^{r}-\tau\left(-L^{\prime}(\tau)\right)^{r} e^{\left(\tau_{n}-\tau\right) y}
$$

Abbreviate the left hand side by $I_{n}$. By a one term Taylor expansion we can write

$$
e^{y\left(\tau_{n}-\tau\right)}=1+y\left(\tau_{n}-\tau\right) e^{y \theta_{n}}
$$

where $-\left(\tau_{n}-\tau\right)_{-} \leq \theta_{n} \leq\left(\tau_{n}-\tau\right)_{+}$. Hence

$$
\begin{aligned}
I_{n}= & \tau_{n}\left(-L_{n}^{\prime}\left(\tau_{n}\right)\right)^{r}-\tau\left(-L^{\prime}(\tau)\right)^{r}-\tau y\left(\tau_{n}-\tau\right)\left(-L^{\prime}(\tau)\right)^{r} e^{y \theta_{n}} \\
= & \left(\tau_{n}-\tau\right)\left(-L^{\prime}(\tau)\right)^{r}+\tau_{n}\left\{\left(-L_{n}^{\prime}\left(\tau_{n}\right)\right)^{r}-\left(-L^{\prime}(\tau)\right)^{r}\right\} \\
& -\tau y\left(\tau_{n}-\tau\right)\left(-L^{\prime}(\tau)\right)^{r} e^{y} \theta_{n}
\end{aligned}
$$


However

$$
\begin{aligned}
\left(-L_{n}^{\prime}\left(\tau_{n}\right)\right)^{r}-\left(-L^{\prime}(\tau)\right)^{r} & =\left[-L_{n}^{\prime}\left(\tau_{n}\right)+L^{\prime}(\tau)\right]\left[\sum_{t=0}^{I-1}\left(-L_{n}^{\prime}\left(\tau_{n}\right)\right)^{t}\left(-L^{\prime}(\tau)\right)^{r-1-t}\right] \\
& =R_{n}\left\{L^{\prime}(\tau)-L_{n}^{\prime}\left(\tau_{n}\right)\right\}
\end{aligned}
$$

where

$$
R_{n}:=\sum_{t=0}^{r-1}\left(-L^{\prime}(\tau)\right)^{r-1-t} S_{1, n}^{t}\left(\tau_{n}\right) \rightarrow r\left(-L^{\prime}(\tau)\right)^{r-1}=: R
$$

almost surely. In turn

$$
\begin{aligned}
& n\left\{L^{\prime}(\tau)-L_{n}^{\prime}\left(\tau_{n}\right)\right\}=\sum_{j=1}^{n}\left(X_{j} e^{-\tau} X_{j}-E\left(X e^{-\tau X}\right)\right) \\
& =\sum_{j=1}^{n}\left(X_{j} e^{-\tau X} j-E\left(X e^{-\tau X}\right)\right)-\left(\tau_{n}-\tau\right) \sum_{j=1}^{n} X_{j}^{2} e^{-\tau(j) X} j
\end{aligned}
$$

by relying on (6). Hence by (3) and (8)

$$
\sqrt{n}\left[L^{\prime}(\tau)-L_{n}^{\prime}\left(\tau_{n}\right)\right]=W_{1, n}(\tau)-\sqrt{n}\left(\tau_{n}-\tau\right) S_{2, n}\left(\tau_{n}, \tau\right)
$$

Combination of (13), (14) and (15) yields

$$
\cdot \sqrt{n} I_{n}=\sqrt{n}\left(\tau_{n}-\tau\right)\left\{\left(1-\tau y e^{y \theta_{n}}\right)\left(-L^{\prime}(\tau)\right)^{r}-\tau_{n} R_{n} S_{2, n}\left(\tau_{n}, \tau\right)\right\}+\tau_{n} R_{n} W_{1, n}(\tau)
$$

We also replace $\sqrt{n}\left(\tau_{n}-\tau\right)$ by its value given by (10). It follows that $\sqrt{n} I_{n}$ is of the form

$$
\sqrt{n} I_{n}=U_{n} W_{0, n}(\tau)+V_{n} W_{1, n}(\tau)
$$


where

$$
\begin{aligned}
& \quad U_{n}=\left\{\left(1-\tau y e^{y \theta_{n}}\right)\left(-L^{\prime}(\tau)\right)^{I}-\tau_{n} R_{n} S_{2, n}\left(\tau_{n}, \tau\right)\right\} / S_{1, n}\left(\tau_{n}, \tau\right) \\
& V_{n}=\tau_{n} R_{n} .
\end{aligned}
$$

By proposition 3, almost surely

$$
\mathrm{U}_{\mathrm{n}} \rightarrow \mathrm{U}:=\left\{(1-\tau \mathrm{y})\left(-\mathrm{L}^{\prime}(\tau)\right)^{\mathrm{r}}-\tau \mathrm{RE}\left(\mathrm{X}^{2} \mathrm{e}^{-\tau \mathrm{X}}\right)\right\} / \mathrm{E}\left[\mathrm{Xe}^{-\tau \mathrm{X}}\right]
$$

and $V_{n} \rightarrow V:=\tau R$ where $R=r\left(-L^{\prime}(\tau)\right)^{r-1}$. Rewrite (16) in the form

$$
\begin{gathered}
\sqrt{n} I_{n}=\frac{1}{\sqrt{n}} \sum_{j=1}^{n}\left[U\left(e^{-\tau X_{j}}-E\left(e^{-\tau X}\right)\right)+V\left(X_{j} e^{-\tau X_{j}}-E\left(X e^{-\tau X}\right)\right)\right] \\
+\left(U_{n}-U\right) W_{0, n}(\tau)+\left(V_{n}-V\right) W_{1, n}(\tau)
\end{gathered}
$$

then proposition 4 can be applied a number of times. We ultimately find

$$
\sqrt{\mathrm{n}} \mathrm{I}_{\mathbf{n}} \stackrel{\mathfrak{D}}{\rightarrow} \mathfrak{N}\left(0, \delta^{2}\right)
$$

where

$$
\delta^{2}=\operatorname{Var}\left[(U+V X) e^{-\tau X}\right]
$$

Returning to $C_{n}-C$ we obtain

$$
\sqrt{\mathfrak{n}}\left(C_{n}-C\right) \stackrel{\mathfrak{D}}{\longrightarrow} \mathfrak{N}\left(0, \delta_{1}^{2}\right)
$$

where

$$
\delta_{1}^{2}=\delta^{2} /\left\{\mathrm{e}^{-\tau \mathrm{y}} \tau^{2}\left(\mathrm{~L}^{\prime}(\tau)\right)^{2 \mathrm{r}}\right\}^{2}
$$

by another application of proposition 4 . 


\section{CHERNOFF BOUNDS}

Assume $\sigma>0$. Let $s<0$. Then for $y \geq 0, L(s)=\int_{0}^{\infty} e^{-s x} d F(x) \geq \int_{y}^{\infty} e^{-s x} d F(x) \geq$ $\mathrm{e}^{-\mathrm{Sy}} \mathrm{P}[\mathrm{X}>\mathrm{y}]$. Hence we get the Chernoff bound

$$
\mathrm{P}[\mathrm{X}>\mathrm{y}] \leq \underset{-\sigma<\mathrm{s}<0}{\inf } \mathrm{e}^{\mathrm{sy}} \mathrm{L}(\mathrm{s})
$$

This means that

$$
\mathrm{P}[\mathrm{X}>\mathrm{y}] \leq \mathrm{e}^{\tau \mathrm{y}_{\mathrm{L}}} \mathrm{L}(\tau)
$$

where

$$
y L(\tau)+L^{\prime}(\tau)=0
$$

(i) The complete monotonicity of $L$ implies that $\frac{-L^{\prime}}{L}$ is decreasing for $s>-\sigma$. Hence $-L^{\prime}(\tau) / L(\tau)>-L^{\prime}(0)=: \mu$ if $\tau<0$. Hence we restrict attention in (17) to $y>\mu$.

(ii) We estimate $\tau$ by solving $\mathrm{yL}_{\mathbf{n}}\left(\tau_{n}\right)+\mathrm{L}_{\mathrm{n}}^{\prime}\left(\tau_{\mathrm{n}}\right)=0$. Now, as before $\tau_{\mathrm{n}} \rightarrow \tau$ a.s.

Furthermore

$$
\begin{aligned}
0 & =\mathrm{n}\left\{\mathrm{yL}_{\mathrm{n}}\left(\tau_{\mathrm{n}}\right)+\mathrm{L}_{\mathbf{n}}^{\prime}\left(\tau_{\mathrm{n}}\right)\right\}-\mathrm{n}\left\{\mathrm{yL}(\tau)+\mathrm{L}^{\prime}(\tau)\right\} \\
& =\mathrm{y} \sum_{\mathrm{j}=1}^{\mathrm{n}}\left(\mathrm{e}^{-\tau \mathrm{n} \mathrm{X}_{\mathrm{j}}}-\mathrm{E}\left(\mathrm{e}^{-\tau \mathrm{X}}\right)\right)-\sum_{\mathrm{j}=1}^{\mathrm{n}}\left(\mathrm{X}_{\mathrm{j}} \mathrm{e}^{-\tau \mathrm{T}_{\mathrm{n}} \mathrm{X}}-\mathrm{E}\left(\mathrm{Xe}^{-\tau \mathrm{X}}\right)\right)
\end{aligned}
$$

By (6) and a little algebra we obtain

$$
\sqrt{n}\left(\tau_{n}-\tau\right)=\frac{\mathrm{yV}_{0, \mathrm{n}}(\tau)-\mathrm{W}_{1, \mathrm{n}}(\tau)}{\mathrm{yS} \mathrm{I}_{1, \mathrm{n}}\left(\tau_{\mathrm{n}}, \tau\right)-\mathrm{S}_{2, \mathrm{n}}\left(\tau_{\mathrm{n}}, \tau\right)} \rightarrow \mathfrak{N}\left(0, \gamma_{1}^{2}\right)
$$


where

$$
\gamma_{1}^{2}=\frac{\operatorname{Var}\left((y-X) e^{-\tau X}\right)}{E^{2}\left(X(y-X) e^{-\tau X}\right)}
$$

As before a consistent estimator for $\gamma_{1}^{2}$ and an asymptotic confidence interval for $\tau_{\mathrm{n}}$ can be constructed.

(iii) We estimate the Chernoff bound $\mathrm{C}:=\mathrm{e}^{\tau \mathrm{y}} \mathrm{L}(\tau)$ by the sample statistic $\mathrm{C}_{\mathrm{n}}:=\mathrm{e}^{\tau_{\mathrm{n}} \mathrm{y}^{\mathrm{y}}} \mathrm{L}_{\mathrm{n}}\left(\tau_{\mathrm{n}}\right)$. As in (11) using (12)

$$
\begin{aligned}
e^{-\tau y}\left(C_{n}-C\right) & =e^{y\left(\tau_{n}-\tau\right)} L_{n}\left(\tau_{n}\right)-L(\tau) \\
& =\left\{L_{n}\left(\tau_{n}\right)-L(\tau)\right\}+L_{n}\left(\tau_{n}\right) e^{y \theta_{n_{y}}\left(\tau_{n}-\tau\right)}
\end{aligned}
$$

Now $\sqrt{n}\left(L_{n}\left(\tau_{n}\right)-L(\tau)\right)=W_{0, n}(\tau)-\sqrt{n}\left(\tau_{n}-\tau\right) S_{1, n}\left(\tau_{n}, \tau\right)$ so that

$$
\sqrt{\mathrm{n}} \mathrm{e}^{-\tau \mathrm{y}}\left(\mathrm{C}_{\mathrm{n}}-\mathrm{C}\right)=\sqrt{\mathrm{n}}\left(\tau_{\mathrm{n}}-\tau\right)\left\{\mathrm{ye} \mathrm{y}^{\mathrm{y} \theta_{\mathrm{n}_{\mathrm{n}}}}\left(\tau_{\mathrm{n}}\right)-\mathrm{S}_{1, \mathrm{n}}\left(\tau_{\mathrm{n}}, \tau\right)\right\}+\mathrm{W}_{0, \mathrm{n}}(\tau)
$$

Introducing (19) into this expression yields after easy algebra

$$
\sqrt{n}\left(C_{n}-C\right)=U_{n} W_{0, n}(\tau)+V_{n} W_{1, n}(\tau)
$$

where

$$
U_{n}=e^{\tau y} \frac{y^{2} e^{y \theta}{ }^{y} L_{n}\left(\tau_{n}\right)-S_{2, n}\left(\tau_{n}, \tau\right)}{y S_{1, n}\left(\tau_{n}, \tau\right)-S_{2, n}\left(\tau_{n}, \tau\right)} \stackrel{\text { a.s. }}{\longrightarrow} e^{\tau y} \frac{y^{2} L(\tau)-L^{\prime \prime}(\tau)}{-y(\tau)-L^{\prime \prime}(\tau)}=: U
$$


and

$$
\mathrm{V}_{\mathrm{n}}=\mathrm{e}^{\tau y} \frac{\mathrm{S}_{1, \mathrm{n}}\left(\tau_{\mathrm{n}}, \tau\right)-\mathrm{ye}{ }^{\mathrm{y} \theta_{\mathrm{n}_{\mathrm{L}}}\left(\tau_{\mathrm{n}}\right)}}{\mathrm{yS}_{1, \mathrm{n}}\left(\tau_{\mathrm{n}}, \tau\right)-\mathrm{S}_{2, \mathrm{n}}\left(\tau_{\mathrm{n}}, \tau\right)} \stackrel{\text { a.s. }}{\longrightarrow} \mathrm{e}^{\tau y} \frac{-\mathrm{L}^{\prime}(\tau)-\mathrm{yL}(\tau)}{-\mathrm{yL}(\tau)-\mathrm{L}^{\prime}(\tau)}=: \mathrm{V}
$$

Note however that by $(18) y^{2} L(\tau)=-y L^{\prime}(\tau)$ so that $U=e^{\tau y}$ while $V=0$. Hence

$$
\sqrt{n}\left(C_{n}-C\right) \stackrel{D}{\longrightarrow} \mathfrak{N}\left(0, e^{2 \tau y} \sigma_{0}^{2}(\tau)\right)
$$

\section{THE CLASSICAL RUIN PROBLEM}

Assume that $\mathrm{F}$ is the distribution of claim sizes $\left\{\mathrm{X}_{\mathrm{j}}\right\}_{1}^{\infty}$ with $\mathrm{EX}=\mu$ in an insurance context where claims arrive according to a Poisson process $\{N(t), t \geq 0\}$ with intensity $\lambda$. Starting with initial reserve $x \geq 0$ and with incoming payments in the time interval $[0, t]$ equal to $t$, the company accumulates the risk reserve

$$
Y(t)=x+t-\sum_{i=1}^{N(t)} X_{i}
$$

The probability of non-ruin with initial reserve $\mathbf{x}$ is then

$$
W(x):=\underset{t>0}{P}\{\inf Y(t)>0\}
$$

It can be shown (see Takács (1967,p.150) Feller (1971,p.377) or Buhlmann $(1970$, p. 144)) that, if the expected claims paid per unit time $\rho:=\lambda \mu<1$, then

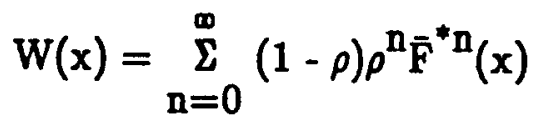

where $\bar{F}$ is the equilibrium distribution corresponding to $F$, i.e.

$$
\bar{F}(x)=\frac{1}{\mu} \int_{0}^{x}[1-F(y)] d y .
$$


Clearly

$$
\overline{\mathrm{L}}(\mathrm{s})=\{1-\mathrm{L}(\mathrm{s})\} / \mu \mathrm{s} .
$$

The famous Lundberg-Cramér ruin estimate is given by

$$
1-W(x) \sim \frac{1-\rho}{\rho|\tau|\left|\bar{L}^{\prime}(\tau)\right|} \mathrm{e}^{\tau \mathrm{x}}, \mathrm{x} \rightarrow \infty
$$

where $\bar{L}(\tau)=\frac{1}{\rho}$.

(i) Note first that $\tau<0$ since $\rho<1$; the existence of $\tau$ clearly depends on the condition $\overline{\mathrm{L}}(-\sigma)>\frac{1}{\rho}$ or $\mathrm{L}(-\sigma)>1+\frac{\sigma}{\lambda}$. We rewrite $(20)$ somewhat. Consider $\mathrm{h}(\mathrm{s}):=$ $s L^{\prime}(s)-L(s)$; then $h^{\prime}(s)=s L^{\prime \prime}(s)$ which is negative for $s<0$. Hence for $\tau<0$, $\mathrm{h}(\tau)=\tau \mathrm{L}^{\prime}(\tau)-\mathrm{L}(\tau)>\mathrm{h}(0)=-1$.

Now in $(20) \overline{\mathrm{L}}^{\prime}(\tau)=\left\{-\tau \mathrm{L}^{\prime}(\tau)-1+\mathrm{L}(\tau)\right\} / \mu \tau^{2}<0$ so that $(20)$ is replaced by

$$
1-W(x) \sim \frac{(1-\rho) \mu}{\rho\left(-L^{\prime}(\tau)-\frac{1}{\lambda}\right)} e^{\tau x}
$$

where we used $\overline{\mathrm{L}}(\tau)=\frac{1}{\rho}$ or $\mathrm{L}(\tau)=1-\frac{\tau}{\lambda}$.

(ii) We estimate $\tau$ by $\tau_{n}$, solution of $L_{n}\left(\tau_{n}\right)=1-\tau_{n} \cdot$ Hence

$$
\lambda\left\{L_{n}\left(\tau_{n}\right)-L(\tau)\right\}=\left(\tau-\tau_{n}\right)
$$

or with (6) and the usual algebra

$$
\sqrt{\mathrm{n}}\left(\tau_{\mathrm{n}}-\tau\right)=\frac{\mathrm{W}_{0, \mathrm{n}}(\tau)}{\mathrm{S}_{1, \mathrm{n}}\left(\tau_{\mathrm{n}}, \tau\right)-\frac{1}{\lambda}} \stackrel{\mathcal{D}}{\longrightarrow} \mathfrak{N}\left(0, \zeta^{2}\right)
$$

where $\zeta^{2}=\frac{\sigma_{0}^{2}(\tau)}{\left(-L^{\prime}(\tau)-\frac{1}{\lambda}\right)^{2}}$ 
(iii) To estimate the probability of non-ruin we put $C=e^{\tau x}\left\{-L^{\prime}(\tau)-\frac{1}{\lambda}\right\}^{-1}$ and $C_{n}=$ $\mathrm{e}^{\tau_{\mathrm{n}} \mathrm{x}}\left\{-\mathrm{L}_{\mathrm{n}}^{\prime}\left(\tau_{\mathrm{n}}\right)-\frac{1}{\lambda}\right\}^{-1}$. Then

$$
I_{n}:=e^{-\tau x}\left[L_{n}^{\prime}\left(\tau_{n}\right)+\frac{1}{\lambda}\right]\left[L^{\prime}(\tau)+\frac{1}{\lambda}\right]\left(C_{n}-C\right)
$$

can be rewritten by (12) in the form

$$
I_{n}=L_{n}^{\prime}\left(\tau_{n}\right)-L^{\prime}(\tau)-\left(\tau_{n}-\tau\right)\left(L^{\prime}(\tau)+\frac{1}{\lambda}\right) x e^{\theta_{n} x}
$$

with the usual operations and (22) we obtain

$$
\sqrt{n} I_{n}=U_{n} W_{0, n}(\tau)-W_{1, n}(\tau)
$$

where

$$
U_{n}=\frac{S_{2, n}\left(\tau_{n}, \tau\right)-\left(L^{\prime}(\tau)+\frac{1}{\lambda}\right) x e^{\theta_{n} x}}{S_{1, n^{n}}\left(\tau_{n^{\prime}}, \tau\right)-\frac{1}{\lambda}} \stackrel{\text { a.s. }}{\longrightarrow} x-\frac{L^{\prime \prime}(\tau)}{L^{\prime}(\tau)+\frac{1}{\lambda}}:=U
$$

Hence

$$
\sqrt{\mathrm{n}}\left(\mathrm{C}_{\mathrm{n}}-\mathrm{C}\right) \stackrel{\mathfrak{D}}{\longrightarrow} \mathfrak{N}\left(0, \zeta_{1}^{2}\right)
$$

where

$$
\zeta_{1}^{2}=\mathrm{e}^{2 \pi x} \operatorname{Var}\left\{(U-X) e^{-\tau X}\right\} /\left[L^{\prime}(\tau)+\frac{1}{\lambda}\right]^{4}
$$

\section{THE COMPOUND POISSON DISTRIBUTION}

Starting with a similar setup as in the previous example one can derive an asymptotic expression for the distribution of the totality of claims incurred up to 
time $t$, i.e. $X(t)=\sum_{i=1}^{N}(t) X_{i}$. Under a number of weak conditions it was shown in Embrechts, Jensen e.a. (1985) that

$$
P\{X(t)>y\} \sim \frac{e^{\tau y}\left[e^{-\lambda t[1-L(\tau)]}-e^{-\lambda t}\right]}{|\tau| \sqrt{2 \pi \lambda t L^{\prime \prime}(\tau)}}, y \rightarrow \infty
$$

where

$$
-\lambda t L^{\prime}(\tau)=y
$$

(i) It is clear that we require $y>\mu \lambda t$ to obtain $\tau<0$. We also write $z=y /(\lambda t)$ for brevity.

(ii) Again $\tau_{n}$ is estimated by the solution of $L_{n}^{\prime}\left(\tau_{n}\right)=-z$. As before

$$
\sqrt{\mathrm{n}}\left(\tau_{\mathrm{n}}-\tau\right)=\frac{\mathrm{v}_{1, \mathrm{n}}(\tau)}{\mathrm{S}_{2, \mathrm{n}}\left(\tau_{\mathrm{n}}, \tau\right)} \stackrel{\mathfrak{D}}{\longrightarrow} \mathfrak{N}\left(0, \xi^{2}\right)
$$

where $\xi^{2}=\frac{\sigma_{1}^{2}(\tau)}{\left(L^{\prime \prime}(\tau)\right)^{2}}$

(iii) Put $C=\frac{\mathrm{e}^{\tau \mathrm{y}}\left(\mathrm{e}^{\lambda t \mathrm{~L}(\tau)}-1\right)}{(-\tau)\left(L^{\prime \prime}(\tau)\right)^{\frac{1}{2}}}$ and $\mathrm{C}_{\mathrm{n}}=\frac{\mathrm{e}^{\tau_{\mathrm{n}} \mathrm{y}}\left(\mathrm{e}^{\lambda t \mathrm{~L}_{\mathrm{n}}\left(\tau_{\mathrm{n}}\right)}-1\right)}{\left(-\tau_{\mathrm{n}}\right)\left(\mathrm{L}_{\mathrm{n}}^{\prime \prime}\left(\tau_{\mathrm{n}}\right)\right)^{\frac{1}{2}}}$ and

$$
I_{n}=\tau \tau_{n}\left(L^{\prime \prime}(\tau) L_{n}^{\prime \prime}\left(\tau_{n}\right)\right)^{\frac{1}{2}} e^{-\tau y}\left(C_{n}-C\right)
$$

We use the identity $\sqrt{a}-\sqrt{b}=(a-b) /(\sqrt{a}+\sqrt{b})$ and a further Taylor expansion

$$
e^{\lambda t L_{n}\left(\tau_{n}\right)}=e^{\lambda t L(\tau)}+\lambda t\left[L_{n}\left(\tau_{n}\right)-L(\tau)\right] e^{\lambda t \Omega n}
$$


where

$$
\min \left(\mathrm{L}_{n}\left(\tau_{n}\right), \mathrm{L}(\tau)\right) \leq \Omega_{\mathrm{n}} \leq \max \left(\mathrm{L}_{\mathrm{n}}\left(\tau_{\mathrm{n}}\right), \mathrm{L}(\tau)\right)
$$

After tedious calculations we arrived at the following expression

$$
\sqrt{n} I_{n}=U_{n} W_{0, n}(\tau)+V_{n} W_{1, n}(\tau)+W_{n} W_{2, n}(\tau)
$$

where

$$
\begin{aligned}
& \mathrm{U}_{\mathrm{n}}:=-\tau \lambda t \mathrm{t}^{\lambda t \Omega_{\mathrm{n}}} \sqrt{L^{\prime \prime}(\tau) \stackrel{\text { a.s. }}{\longrightarrow}}-\tau \lambda t \mathrm{e}^{\lambda t \mathrm{~L}(\tau)} \sqrt{L^{\prime \prime}(\tau)}=: \mathrm{U}, \\
& \mathrm{V}_{\mathrm{n}}:=\left\{\mathrm{S}_{2, \mathrm{n}}\left(\tau_{\mathrm{n}}, \tau\right)\right\}^{-1}\left\{\sqrt{L^{\prime \prime}(\tau)}\left[\left(\mathrm{e}^{\lambda t L(\tau)}-1\right)\left(1-\tau \mathrm{ye} \mathrm{y}^{\mathrm{y} \theta_{\mathrm{n}}}\right)+\tau \lambda t \mathrm{e}^{\lambda t \Omega_{\mathrm{n}}} \mathrm{S}_{1, \mathrm{n}}\left(\tau_{\mathrm{n}}, \tau\right)\right]\right. \\
& -\frac{-\tau_{n}\left(e^{\lambda t L(\tau)}-1\right)}{\sqrt{L_{n}^{\prime \prime}\left(\tau_{n}\right)}+\sqrt{L^{\prime \prime}(\tau)}} S_{3, n}\left(\tau_{n}, \tau\right) \\
& \left.-\frac{\sqrt{L^{n}(\tau)}}{\sqrt{n}} \tau \lambda \text { tye }^{\mathrm{y} \theta_{\mathrm{n}}+\lambda t \Omega_{\mathrm{n}}}\left(\mathrm{W}_{0, \mathrm{n}}(\tau)-\sqrt{\mathrm{n}}\left(\tau_{\mathrm{n}}-\tau\right) \mathrm{S}_{1, \mathrm{n}}\left(\tau_{\mathrm{n}}, \tau\right)\right)\right\} \\
& \text { a.s. } \frac{1}{L^{\prime \prime}(\tau)}\left\{\left(\mathrm{e}^{\lambda t \mathrm{~L}(\tau)}-1+\tau \mathrm{y}\right) \sqrt{L^{\prime \prime}(\tau)}-\frac{1}{2}\left(\mathrm{e}^{\lambda t \mathrm{~L}(\tau)}-1\right) \frac{\tau \mathrm{L}^{(3)}(\tau)}{\sqrt{L^{\prime \prime}(\tau)}}\right\}=: \mathrm{V} \text {, }
\end{aligned}
$$

using (24), and

$$
W_{n}:=\frac{\tau_{n}\left(e^{\lambda t L(\tau)}-1\right)}{\sqrt{L_{n}^{\prime \prime}\left(\tau_{n}\right)}+\sqrt{L^{\prime \prime}(\tau)}} \frac{\text { a.s. }}{\tau} \frac{\tau\left(e^{\lambda t L(\tau)}-1\right)}{2 \sqrt{L^{\prime \prime}(\tau)}}=: W
$$

As before we arrive at

$$
\sqrt{n}\left(C_{n}-C\right) \stackrel{\mathfrak{D}}{\longrightarrow} \mathfrak{N}\left(0, \xi_{1}^{2}\right)
$$


where

$$
\xi_{1}^{2}=\left(\frac{\mathrm{e}^{\tau \mathrm{y}}}{\tau^{2} \mathrm{~L}^{\prime \prime}(\tau)}\right)^{2} \operatorname{Var}\left\{\left(\mathrm{U}+\mathrm{VX}+\mathrm{WX} \mathrm{X}^{2}\right) \mathrm{e}^{-\tau \mathrm{X}}\right\}
$$

\section{THE COMPOUND PÓLYA DISTRIBUTION}

In Embrechts, Jensen e.a. (1985) the compound Pólya distribution was approximated as well; here

$$
P\{X(t) \leq x\}=\sum_{n=0}^{\infty}\left(\begin{array}{c}
k+n-1 \\
n
\end{array}\right)\left(\frac{t}{t+k}\right)^{n}\left(\frac{k}{t+k}\right)^{n} F^{* n}(x)
$$

where $\mathbf{k}>1$.

Under weak conditions it was shown that

$$
\left.\left.P[X(t)>y] \sim \frac{\mathbf{k}^{\mathbf{k}} \mathrm{e}^{-\mathbf{k}}}{\Gamma(\mathbf{k})} \frac{\mathrm{e}^{\tau \mathbf{y}}}{\Gamma \tau \mid \mathrm{y}}\left\{\left(\frac{\mathbf{y}}{-\mathrm{tL}^{\prime}(\tau)}\right)^{\mathbf{k}}-\left(\frac{\mathbf{k}}{\mathrm{t}+\mathrm{k}}\right)\right)\right)^{\mathbf{k}}\right\}, \quad \mathrm{y} \rightarrow \infty
$$

where

$$
-t L^{\prime}(\tau)=y\left\{1+\frac{t}{k}[1-L(\tau)]\right\}
$$

(i) Since $\mathrm{y} \rightarrow \infty$ we can assume that $\mathrm{y}>\mu$ t so that the increasing function $1+\frac{t}{k}[1-L(s)]$ (with value 1 at $s=0$ ) intersects the decreasing function $-\frac{t}{y} L^{\prime}(s)$ (with value $\frac{\mu \mathrm{t}}{\mathrm{y}}$ at $\mathrm{s}=0$ ) at some value $\tau<0$.

We simplify the notation somewhat by introducing the constants

$$
z=\frac{y}{t}, a=\left(\frac{k}{t+k}\right)^{k}, b=\frac{y}{k}
$$

(ii) Again $\tau_{n}$ is defined by $-L_{n}^{\prime}\left(\tau_{n}\right)=z+b\left[1-L_{n}\left(\tau_{n}\right)\right\}$ where $\left.-L^{\prime}(\tau)=z+b[1-L(\tau)]\right\}$ defines $\tau$. Hence elimination of $\mathrm{z}$ yields 


$$
L^{\prime}(\tau)-L_{n}^{\prime}\left(\tau_{n}\right)=b\left\{L(\tau)-L_{n}\left(\tau_{n}\right)\right\}
$$

By now the calculations are routine; we obtain

$$
\sqrt{n}\left(\tau_{n}-\tau\right)=\frac{W_{1, n}(t)+b W_{0, n}(t)}{S_{2, n}\left(\tau_{n}, \tau\right)+b S_{1, n}\left(\tau_{n}, \tau\right)} \stackrel{D}{\longrightarrow} \mathfrak{N}\left(0, K^{2}\right)
$$

where

$$
\mathrm{K}^{2}=\operatorname{Var}\left\{(\mathrm{X}+\mathrm{b}) \mathrm{e}^{-\tau \mathrm{X}}\right\} / \mathrm{E}^{2}\left\{\mathrm{X}(\mathrm{X}+\mathrm{b}) \mathrm{e}^{-\tau \mathrm{X}}\right\}
$$

(iii) Put $C=\frac{e^{\tau y}}{|\tau|}\left\{\left(\frac{z}{-L^{\prime}(\tau)}\right)^{k}-a\right\}, C_{n}=\frac{e^{\tau} \mathbf{n}^{y}}{\left|\tau_{n}\right|}\left\{\left(\frac{z}{-L_{n}^{\prime}\left(\tau_{n}\right)}\right)^{k}-a\right\}$, and

$$
I_{n}=e^{-\tau y_{n}}\left(C_{n}-C\right)
$$

Again, very much like in the Pascal case, we find

$$
\sqrt{\mathrm{n}} \mathrm{I}_{\mathrm{n}}=\mathrm{U}_{\mathrm{n}} \mathrm{W}_{0, \mathrm{n}}(\tau)+\mathrm{V}_{\mathrm{n}} \mathrm{W}_{1, \mathrm{n}}(\tau)
$$

where

$$
\begin{aligned}
\frac{1}{b} U_{n}\left\{S_{2, n}\left(\tau_{n}, \tau\right)+\right. & \left.b S_{1, n}\left(\tau_{n^{\prime}}, \tau\right)\right\}:=\left[\left(\frac{-z}{L^{\prime}(\tau)}\right)^{k}-a\right]-y \tau e^{y \theta}\left[\left(\frac{-z}{L^{\prime}\left(\tau_{n}\right)}\right)^{k}-a\right] \\
+ & S_{2, n^{\prime}}\left(\tau_{n^{\prime}}, \tau\right) \frac{\tau(-z)^{k}}{\left[L^{\prime}(\tau) L_{n}^{\prime}\left(\tau_{n}\right)\right]^{k}} \sum_{m=0}^{k-1}\left(L_{n}^{\prime}\left(\tau_{n}\right)\right)^{m^{m}\left(L^{\prime}(\tau)\right)^{k-1-m}} \\
& \stackrel{a . s .}{\longrightarrow}(1-y \tau)\left[\left(\frac{-z}{L^{\prime}(\tau)}\right)^{k}-a\right]-\frac{k \tau z^{k} L^{\prime \prime}(\tau)}{\left[-L^{\prime}(\tau)\right]^{k+1}} \\
& =: \frac{1}{b} U E\left[X(X+b) e^{-\tau X}\right]
\end{aligned}
$$


and

$$
\begin{aligned}
& \mathrm{V}_{\mathrm{n}}\left\{\mathrm{S}_{2, \mathrm{n}}\left(\tau_{\mathrm{n}}, \tau\right)+\mathrm{bS}_{1, \mathrm{n}}\left(\tau_{\mathrm{n}}, \tau\right)\right\}:=\left[\left(\frac{-\mathrm{z}}{\Gamma^{\prime}(\tau)}\right)^{\mathbf{k}}-\mathrm{a}\right]-\mathrm{y} \tau \mathrm{e}^{\mathrm{y} \theta_{\mathrm{n}}}\left[\left(\frac{-\mathrm{z}}{L_{\mathrm{n}}^{\prime}\left(\tau_{\mathrm{n}}\right.}\right)^{\mathrm{k}}-\mathrm{a}\right] \\
& -\mathrm{bS}_{1, \mathrm{n}}\left(\tau_{\mathrm{n}^{\prime}}, \tau\right) \frac{\tau(-\mathrm{z})^{\mathrm{k}}}{\left[\mathrm{L}^{\prime}(\tau) \mathrm{L}_{\mathrm{n}}^{\prime}\left(\tau_{\mathrm{n}}\right)\right]^{\mathrm{k}}} \sum_{\mathrm{m}=0}^{\mathrm{k}} \sum^{-1}\left(\mathrm{~L}_{\mathrm{n}}^{\prime}\left(\tau_{\mathrm{n}}\right)\right)^{\mathrm{m}}\left(\mathrm{L}^{\prime}(\tau)^{\mathrm{k}-1-\mathrm{m}}\right. \\
& \stackrel{\text { a.s. }}{\longrightarrow}(1-\mathrm{y} \tau)\left[\left(\left(_{\Gamma^{\prime}(\tau)}^{-\mathrm{z}}\right)^{\mathrm{k}}-\mathrm{a}\right]+\frac{\mathrm{kb} \tau \mathrm{z}^{\mathrm{k}}}{\left[-\mathrm{L}^{\prime}(\tau)\right]^{\mathrm{k}}}=: \mathrm{VE}\left[\mathrm{X}(\mathrm{X}+\mathrm{b}) \mathrm{e}^{-\tau \mathrm{X}_{1}}\right]\right. \text {. }
\end{aligned}
$$

Hence

$$
\sqrt{\mathrm{n}}\left(\mathrm{C}_{\mathrm{n}}-\mathrm{C}\right) \stackrel{\mathfrak{D}}{\longrightarrow} \mathfrak{N}\left(0, \mathrm{~K}_{1}^{2}\right)
$$

where

$$
\mathrm{K}_{1}^{2}=\mathrm{e}^{2 \tau \mathrm{y}} \tau^{-4} \operatorname{Var}\left\{(\mathrm{U}+\mathrm{VX}) \mathrm{e}^{-\tau \mathrm{X}}\right\}
$$

\section{REMARKS}

As mentioned in the summary, the relationship

$$
P\left\{\sum_{i=1}^{N} X_{i}>y\right\} \sim \varphi\left(y, \tau, L(\tau), L^{\prime}(\tau), \ldots\right)=: \varphi
$$

where $\psi(\tau, L(\tau), \ldots)=0$, holds for $\mathbf{y} \rightarrow \boldsymbol{w}$. On top of this first approximation we replaced the right hand side of (25) by

$$
\varphi_{\mathrm{n}}:=\varphi\left(\mathrm{y}, \tau_{\mathbf{n}}, \mathrm{L}_{\mathrm{n}}\left(\tau_{\mathrm{n}}\right), \mathrm{L}_{\mathbf{n}}^{\prime}\left(\tau_{\mathrm{n}}\right), \ldots\right)
$$

where $L_{n}$ was the empirical version of $L$ and where $\tau_{n}$ was the sample version of the solution of the equation $\psi\left(\tau_{n}, L_{n}\left(\tau_{n}\right), \ldots\right)=0$. The reader could wonder how accurate the superimposed approximations will be. 
To evaluate the accuracy of (25) in itself is an important but hard problem. In some cases the proof of (25) is easy, like in the Chernoff bounds; however more typically the proof depends on deep theorems from the theory of stochastic processes or on intricate procedures from asymptotic analysis. Hence a second order term or even a series expansion would be desirable. Let us remark that for the compound Pólya and for the compound Poisson case, second and higher order terms are available in Embrechts, Jensen e.a. (1985).

A systematic study of the accuracy of the empirical version $\varphi_{n}$ in evaluating $\varphi$ is an entirely different issue, depending heavily upon simulation studies. As a simple test case we evaluated the Chernoff bound for the exponential distribution with mean 1 . Then $\mathrm{L}(\mathrm{s})=(1+\mathrm{s})^{-1}$; given $\mathrm{y}>1, \tau=\mathrm{y}^{-1}-1$ and $\mathrm{C}=\mathrm{e}^{\tau y} \mathrm{~L}(\tau)=\mathrm{y} \mathrm{e} \mathrm{e}^{1-\mathrm{y}}$; also $\mathrm{I}=(-1, \infty)$. After substantial simulation we could only conclude that the problem is far from trivial. For small $y$-values the accuracy was always found to be sufficient even though we encountered systematic bias; for large values of $y$ however the accuracy is far less satisfying.

Here are some of our temporary conclusions :

(i) In many examples $L(s)$ and its derivatives have a vertical asymptote at $s=\sigma$; as a result the solution of the implicit equation $\psi\left(\tau_{n}, L_{n}\left(\tau_{n}\right), \ldots\right)=0$ for $\tau_{n}$ induces systematic bias. Also the numerical procedure (bisection, Newton-Raphson,...) influences the accuracy but to a lesser extent.

(ii) In all of our examples we made heavy use of proposition 2; a requirement for its applicability was however that $2 \tau>\sigma$. In our simulation example, y values greater than 2 caused large fluctuations in the estimation of $\tau$ and C. Moreover, the larger $y$, the closer we get to the singularity of the function $L(s)$.

(iii) Presumably the most important reason for the eventual poor behavior of the sample version $\tau_{n}$ is this : the empirical functions $L_{n}(s)$ are determined by the positive sample values $\mathrm{X}_{1}, \mathrm{X}_{2}, \ldots, \mathrm{X}_{\mathrm{n}}$; in all our formulae however we use the extrapolated values of $L_{n}(s)$ for $s<0$; moreover the latter function is entire for every $n$ while $L(s)$ has a singularity at $\sigma<0$. 


\section{ACKNOWLEDGEMENT}

We take pleasure in thanking C. Coleman for extensive simulation work that unfortunately could not be incorporated in this paper. Also thanks are due to W. Van Assche and to a referee for some helpful comments.

Part of this work was done while the second author was on sabbatical leave at the Statistics and Applied Probability Program at the University of California, Santa Barbara. The author extends his gratitude to J. Gani and UCSB for the kind hospitality.

\section{REFERENCES}

BEEKMAN, J.A. (1974) : Two Stochastic Processes, Almquist \& Wiksell, Stockholm.

BUHLMANN, H. (1970) : Mathematical Methods of Risk Theory, Springer Verlag, Berlin.

CSÖRGÖ, S. (1982) : The empirical moment generating function. Coll. Math. Soc. J. Bolyai, 32, Nonparametric statistical inference, B.V. Gnedenko, M.L. Puri \& I. Vincze ed., Amsterdam, 139-150.

EMBRECHTS, P., MAEJIMA, M. \& TEUGELS, J.L. (1985) : Asymptotic behaviour of compound distributions, Astin Bulletin, $\underline{15}$, 45-48.

EMBRECHTS, P., JENSEN, J.L., MAEJIMA, M. \& TEUGELS, J.L. (1985) : Approximations for compound Poisson and Pólya processes, Adv. Appl. Probability, 17, 623-637.

FELLER, W. (1977) : An Introduction to Probability Theory and its Applications, Vol. II, ed, J. Wiley \& Sons, New York.

SERFLING, R.J. (1980) : Approximation Theorems of Mathematical Statistics, J. Wiley \& Sons, New York. 
SUNDT, B. (1982) : Asymptotic behaviour of compound distributions and stop loss premiums, Astin Bulletin, $\underline{13}, 89-98$.

TAKÁCS, L. (1967) : Combinatorial Methods in the Theory of Stochastic Processes, J. Wiley \& Sons, New York.

TEUGELS, J.L. (1985) : Approximation and estimation of some compound distributions, Insurance : Math. \& Econ., $\underline{4}, 143-153$. 